

\section{INDICE}

EDITORIAL PERSONAL ÁMBITOS

Apresentação do monográfico. Abordagem qualitativa: olhares e práticas transdisciplinares nas ciências antropossociais

Presentation of the monograph. Qualitative approach: transdisciplinary views and practices in anthroposocial sciences

Ronaldo Nunes Linhares, António Pedro Costa

MONOGRAFICOS MONOGRAPHS

Identidades femininas na rede: as crianças falam!

Female identities on line: children can speak

Marta Maria Azevedo Queiroz

$12-31$

Transição de cuidados de enfermagem: ISBAR na promoção da segurança dos

doentes - revisão scoping

Transition of nursing care: ISBAR in promoting patient safety - scoping review

Ana Rita Esteves Figueiredo, Teresa Maria Ferreira dos Santos Potra, Pedro Ricardo Martins Bernardes Lucas

$32-48$

Integración de elementos cualitativos y cuantitativos en metodología observacional

Integration of qualitative and quantitative elements in observational methodology

M. Teresa Anguera, Angel Blanco-Villaseñor, José Luis Losada, Pedro Sánchez-Algarra

49-70

Atos educativos com oficines de ecografias: uma investigação otobiográfica

Educational acts at echographie's cineliers: an otobiographic research

Silas Borges Monteiro, Anaise Avila Severo

Actuación de las políticas: política como texto y política como discurso

Action of policies: policy as text and policy as discourse

Mónica Rocío Barón

88-104 


\section{ÁMBITOS PERSONALES PERSONAL ÁMBITOS}

Un retrato de la cultura local a través del Periodismo cultural. Análisis comparado de Sevilla y Porto Alegre

A portrait of the local culture through cultural Journalism. Comparative analysis of Seville and Porto Alegre

\section{ARTÍCULOS ARTICLES}

Microsociología del profesor universitario

Microsociology of an university professor

Antonio Fernández Vicente

La pobreza y el discurso de los mass media. Un estudio de la prensa local argentina

Poverty and mass media 's discourse. A study of the Argentine local press

María del Rosario Sanchez, Silvia London

La comunicación no verbal en las elecciones andaluzas de 2018. Comparativa de Susana Díaz y Teresa Rodríguez en el debate de RTVE

Non-verbal communication in the Andalusian municipal elections of 2018. Comparison of Susana Díaz and Teresa Rodríguez in the electoral RTVE debate

María Hernández Herrarte, Patricia Zamora-Martínez

El infoentretenimiento en la televisión de pago, Movistar+ y el canal \#0.

El uso transmedia de sus contenidos de humor

Infotainment on pay television, Movistar+ and channel \# 0 . The transmedia use of its humorous content

Metodología y formación docente cuestiones claves para la integración de las TIC en la educación

Methodology and teacher training as a key issue for ICTs integration in Education 


\section{RESEÑAS REVIEWS}

Aquelarre. Mujeres en la cultura de masas

Coven. Women in mass culture

Regla Ismaray Cabreja Piedra

216-220

Transición ecosocial y principios éticos en el periodismo: una guía para la comunicación de nuevas narrativas

The Eco-social transition and ethical principles in journalism: a guide for the communication of new narratives

Amanda Salazar Torres

Narrativas ecofeministas y mapa de transición ecosocial para medios de comunicación Eco-feminist narratives and ecosocial transition map for the media 


\title{
Integración de elementos cualitativos y cuantitativos en metodología observacional
}

\author{
Integration of qualitative and quantitative elements in \\ observational methodology
}

\author{
M. Teresa Anguera, Universidad de Barcelona, España \\ Facultad de Psicolog.a, Campus Mundet, $P^{\circ}$ Vall d'Hebr. n, 171, 08035, Barcelona \\ mtanguera@gmail.com | Orcid: http://orcid.org/0000-0001-7147-2927 \\ Angel Blanco-Villaseñor*, Universidad de Barcelona, España \\ (D.E.P.) \\ José Luis Losada, Universidad de Barcelona, España \\ Facultad de Psicología, Campus Mundet, Po Vall d'Hebrón, 171, 08035, Barcelona \\ jlosada@ub.edu | Orcid: https://orcid.org/0000-0001-6134-1819 \\ Pedro Sánchez-Algarra, Universidad de Barcelona, España \\ Facultad de Biologia, Diagonal, 643, 08028 Barcelona \\ psanchez@ub.edu | Orcid: http://orcid.org/0000-0001-7739-2709
}

DOI: https://dx.doi.org/10.12795/Ambitos.2020.i49.04 


\title{
Resumen
}

La metodología observacional, a lo largo de las cuatro últimas décadas, se ha consolidado como método científico, y progresivamente se han ido incorporando desarrollos procedimentales que la han dotado de una gran rigurosidad, sin menoscabo de la flexibilidad que le es inherente. En el marco actual de los mixed methods, el aspecto crucial por antonomasia es la integración de elementos cualitativos y cuantitativos, cuestión nada fácil, y sobre la cual se han pronunciado numerosos investigadores relevantes. En este trabajo, de carácter procedimental, se hace una propuesta acerca de la metodología observacional, considerada como mixed method en sí misma, que requieren el desarrollo de fórmulas de integración (quantitizing) entre elementos cualitativos y cuantitativos, y se recomienda la incorporación del análisis bayesiano a los estudios mixed methods, utilizando el investigador su propia experiencia y conocimiento como punto de partida. La vía propuesta de quantitizing toma como punto de partida la realización de una observación sistemática en la cual se obtienen registros descriptivos (fase QUAL); a partir del marco teórico y pericia empírica se construye un instrumento de observación, con una estructura definida, que posibilita la sistematización del registro basándose en el parámetro orden o secuencia, y posibilitará que el registro adopte el formato de una matriz de códigos, y ésta se podrá analizar mediante técnicas cuantitativas (fase QUAN); los resultados se interpretarán retornando al problema inicial de estudio (fase QUAL). La estrategia que conlleva esta forma de realizar el quantitizing es innovadora, y no tiene límites de aplicabilidad.

\begin{abstract}
The observational methodology has consolidated as scientific method along four last decades, and during this period has developed the procedure, with the addition of new advances concerning it. This progress implies the maintenance of two characteristic features: rigor and flexibility, that are essential in this methodology. In the current framework of mixed methods, the core is the integration ways between qualitative and quantitative elements, that is not easy, and prestigious researchers have written about this issue. In this work, focused in procedural aspects, we do a proposal about observational methodology considered itself as mixed method, that imply integration ways (quantitizing) between qualitative and quantitative elements; also, we recommend to set up the bayesian approach to mixed methods studies, that is based on the subjective perspective of probability, and for this the researcher uses his/her own experience and knowledge as a starting point. The proposal of quantitizing begins with a systematic observation, and implies qualitative records (QUAL step); from theoretical framework and empirical expertise it is necessary to build an observation instrument with a specific structure that allows for systematizing the record from order or sequence parameter, and following a matrix codes format, that we will analyze quantitatively (QUAN step); the results will interpret coming back to initial problem (QUAL step). The strategy that implies this quantitizing way is innovative, and it has not applying limits.
\end{abstract}


Palabras clave: Observación sistemática, mixed methods, quantitizing, análisis bayesiano

Keywords: Systematic observation, mixed methods, quantitizing bayesian approach

\section{INTRODUCCIÓN}

En las últimas cuatro décadas, la metodología observacional ha experimentado una profunda transformación, habiéndose consolidado como método científico. Nada tiene que ver la debilidad y simpleza de los estudios iniciales, en que unas pocas hojas con anotaciones era el único "capital" informativo del cual se disponía, sin establecer criterios de ningún tipo, y en donde lo único que cabía era comentarlas e interpretarlas, con los estudios observacionales actuales, totalmente fortalecidos desde los planteamientos del método científico, caracterizados por la difícil combinación entre la flexibilidad y la rigurosidad, y que se publican en revistas con factor de impacto y alto prestigio.

El desarrollo metodológico ha sido imparable, y en la actualidad ha alcanzado un reconocimiento que difícilmente era previsible. Pero el esfuerzo ha de ser constante, y desde el inicio de este siglo y milenio nos hallamos ante un "tercer paradigma", como se ha llamado a los mixed methods, que nos apremia planteándonos un reto sumamente relevante.

El crecimiento de los mixed methods como ámbito de conocimiento ha sido tan rápido en los últimos años que se han producido algunas brechas que siguen abiertas, producidas esencialmente, a nuestro entender, por una crisis de crecimiento, que deberá saldarse con un nuevo paso adelante una vez se resuelvan las múltiples preguntas que siguen formulándose los investigadores. La mera denominación mixed methods tiene un elevado poder de convocatoria en la comunidad científica, habiéndose incrementado notablemente las publicaciones en los últimos años, y creado una atmósfera muy favorable para incentivar el interés indudable que presentan para la investigación en todos los ámbitos.

El estado de la cuestión nos obliga a estar vigilantes y dar un paso adelante. En efecto, por una parte, los trabajos clásicos en mixed methods vuelven a retroceder respecto a la entidad de la observación como tal, sea distinguiendo entre observación cualitativa (observación participante) y observaciòn cuantitativa (en la cual se graba mediante video y se calcula la concordancia), como hacen Johnson y Turner (2003), o Teddie y Tashakkori (2009), que se refieren a tres tipos de "técnicas observacionales" (p. 219), denominadas OBS-QUAL (observación cualitativa), OBS-MM (observación mixed methods), y OBS-QUAN (observacion cuantitativa), que nos obligan a retrotraernos a significados y acepciones actualmente superados, pues por 
observación cuantitativa se considera las respuestas a inventarios o a rating scales, y en ninguna de ellas se arbitra una vía para el análisis de la información.

Y, por otra parte, se ha producido una evolución conceptual de los mixed methods, al expandirse de forma tan rápida, como lo demuestran las 19 definiciones de prestigiosos autores que se recogen en Johnson, Onwuegbuzie y Turner (2007), o los numerosos intentos que tratan de establecer vías que lo posibiliten, o las vanas pretensiones de búsqueda de la simetría entre la vertiente cualitativa y cuantitativa (Anguera, Camerino, Castañer, Sánchez-Algarra, \& Onwuegbuzie, 2017).

Como consecuencia, nuestro objetivo consiste en impulsar un avance para la metodología observacional, y en la cual se aplicará una de las vías de integración (connect) propuestas por Cresswell and Plano Clark (2011), pero con la finalidad de que los datos cualitativos se transformen en otros también cualitativos, aunque estructurados de determinada manera, y a partir de los cuales será posible realizar análisis cuantitativos robustos (Anguera, Blanco-Villaseñor, Losada, y SánchezAlgarra, 2019). De esta manera se conforma nuestra propuesta de quantitizing como vía de integración. Además, con el matiz añadido de que la experiencia y pericia del investigador coadyuvarán a que se alcance la integración total mediante el análisis bayesiano, que aún se halla en fase desarrollo en los mixed methods, pero que ya empieza a dar sus frutos (Anguera, Castañer, Portell, Sánchez-Algarra, \& Camerino, in press; Fuster-Parra, García-Más, Cantallops, Ponseti, \& Luo, 2016; Fuster-Parra, García-Más, Ponseti, \& Leo, 2015; Fuster-Parra, García-Más, Ponseti, Palou, \& Cruz, 2013; Olmedilla, Rubio, Fuster-Parra, Pujals, \& García-Más, 2018).

\section{REVISIÓN TEÓRICA Y PLANTEAMIENTO DEL PROBLEMA}

En este trabajo, que no es un estudio empírico, sino de propuesta metodológica, nos interesa aportar elementos que faciliten la integración entre elementos cualitativos y cuantitativos en estudios de observación sistemática, coadyuvando a que quede definitivamente superado el planteamiento unilateral que se adoptaba en las investigaciones cualitativas, en aras a dotarlas de nuevos elementos enriquecedores. Hasta épocas relativamente recientes, la observación no estaba estructurada como metodología, y la mayoría de las publicaciones sobre metodología cualitativa la incluían entre los diversos tipos de estudios, dado que se consideraba que tenía este carácter; además, tenía escaso poder de convocatoria, y apenas se le dedicaba atención. Podemos recordar trabajos que reflejan la situación en que se hallaba entonces la observación sistemática (Anguera, 1979; Hutt \& Hutt, 1974; Martin \& Bateson, 1991; Mucchielli, 1974; Weick, 1968).

El objetivo de los estudios observacionales es el análisis de la realidad perceptible, en estudios en los cuales se respeta la espontaneidad del comportamiento y la habitualidad del contexto -que caracterizan a la observación sistemática-, y en los 
cuales no existe elicitación de respuesta. Durante décadas se ubicaron en una metodología inicialmente cualitativa para captar narrativamente la realidad tal cual se producía, y fue posteriormente, a partir de los años ochenta y noventa, en que se trabajó intensamente para avanzar en la propuesta de los diseños observacionales, la construcción de instrumentos a medida, la sistematización del registro, la obtención de parámetros, el control de calidad del dato, y el análisis cuantitativo en función del diseño adecuado en cada estudio.

Nos podemos preguntar en qué medida la metodología observacional se ajusta a la caracterización de la metodología cualitativa en una primera fase del proceso y a la aplicación de la cuantitativa en una segunda fase del mismo, y ya nos planteábamos esta pregunta justo a inicios de siglo (Anguera, 2000). Elegimos dos autores como botón de muestra, al margen de que ésta ha sido siempre también nuestra posición: Por una parte, Bakeman y Gottman (1989) se pronuncian taxativamente definiéndola como una forma particular de cuantificar la conducta, y, en efecto, la cuantifican y analizan con rigor, pero ellos mismos dedican varios capítulos de su obra a la explicación y ejemplificación de registros, así como a su posterior codificación, momento de inflexión que permitirá el encaje entre lo cualitativo y lo cuantitativo. Y, por otra parte, Blanco-Villaseñor (1997), en un trabajo titulado Metodologías cualitativas en la investigación psicológica, desarrolla el procedimiento propio de la metodología observacional, insistiendo, una vez se ha llegado a la codificación del registro, en el muestreo de conductas, en el significado de la fiabilidad, precisión y validez para garantizar la calidad del registro, y en el análisis cuantitativo de los registros e investigación de patrones de conducta, cuestiones que no podrían materializarse desde una metodología cualitativa.

Asimismo, trabajos orientados a lograr una adecuada estructuración de los estudios observacionales (Anguera, Blanco-Villaseñor, y Losada, 2001; Anguera, BlancoVillaseñor, Losada, y Portell, 2018; Portell, Anguera, Chacón-Moscoso, \& SanduveteChaves, 2015; Sánchez-Algarra \& Anguera, 2013) coinciden, cada uno desde sus propios matices, en que el procedimiento vertebrador de la metodología observacional está formado por sucesivas macroetapas, de carácter cualitativo primero, y cuantitativo después, para volver de nuevo a la etapa final, también cualitativa, de interpretación de resultados. No debemos desaprovechar en absoluto este planteamiento, que se abre camino en un panorama en que numerosos autores han contribuido a proponer estrategias para integrar datos mediante el análisis, y no tanto a elaborar conclusiones a partir de los resultados del análisis (Bazeley, 2017; Creswell \& Plano Clark, 2017; Teddlie \& Tashakkori, 2009), lo cual no está exento de polémica, en buena medida debido a la existencia de matices diferenciados entre los autores, así como a la existencia de diferentes planteamientos conceptuales y metodológicos. 
Nuestro punto de partida básicamente consta de datos procedentes de observación directa (registros de conducta en situaciones naturales en que se producen comportamientos de forma espontánea, y siempre que se garantice la perceptividad del comportamiento -antes era perceptibilidad visual, pero en la actualidad se substituye por la grabación videográfica, que garantiza una precisión y agilidad mucho mayores-), o de observación indirecta (textos procedentes de la grabación magnética digital de conversaciones formales e informales, discusiones de grupo, entrevistas en profundidad, grupos focales, o también información que originariamente tenía el formato de texto, como diarios, notas manuscritas, informes, etc.). Tanto la observación directa como indirecta presentan una elevada aplicabilidad en prácticamente todas las áreas psicológicas, lo cual nos garantiza una amplia cobertura en cuanto a disponibilidad de información.

En numerosos ámbitos se requiere la información obtenida mediante la observación. Si estudiamos, por ejemplo, cualquier flujo comunicativo (terapeuta y paciente, profesor-alumno, entrenador-deportista, diálogo entre iguales, interacción a nivel intrafamiliar, en las relaciones laborales, etc.), y uno de los interactuantes realiza una determinada acción, aunque posteriormente lo niegue, o diga lo contrario, se estaría contraponiendo la información visual de la realidad registrada, de los episodios ocurridos, con la mediatización interpretativa de un discurso posterior, que además podría estar saturada de un importante bagaje cognitivo. ¿De qué serviría, en una competición deportiva, que un deportista afirmara que realizó determinada acción (por ejemplo, que no tocó a su adversario), si se dispone de una grabación videográfica que nos indica lo contrario?

El volumen, calado y matices de las discrepancias entre las imágenes o sonidos de la realidad y la respuesta a preguntas elicitadas sobre esta realidad (como serían las respuestas a cuestionarios, o entrevistas sistematizadas, o la pasación de tests psicológicos) puede ser considerable. Incluso más, pues esta elicitación de la respuesta no la necesitamos, es totalmente sobrante, dado que la cuestión clave radica en que necesitamos disponer de la riqueza que suponen los fragmentos de la propia realidad transcurrida, por la objetivación que podemos obtener, y no en lo que se comente, describa, valore o interprete acerca de dicha realidad, que tendrá posiblemente una fuerte carga de subjetividad.

Podríamos seguir añadiendo múltiples ejemplos. En todos ellos, la solución únicamente puede venir del desarrollo optimizado de las sucesivas etapas de la metodología observacional, que son las propias del método científico. La observación directa es el modo primario de recogida de datos en el análisis de conductas y de eventos, y se dispone de múltiples evidencias sobre sus grandes posibilidades, así como de una ya larga experiencia de utilización (Anguera, 1979, 2003; Anguera et al., 2001; Anguera, Blanco-Villaseñor, Losada, y Portell, 2018; Chacón-Moscoso et al., 2019; Portell, Anguera, Chacón-Moscoso, et al., 2015; Portell, Anguera, Hernández- 
Mendo, \& Jonsson, 2015a, 2015b; Sánchez-Algarra \& Anguera, 2013). Y recientemente se está desarrollando la observación indirecta (Anguera, 2020, en prensa; Anguera, Portell, Chacón-Moscoso, \& Sanduvete-Chaves, 2018; Krippendorf, 2013; Morales-Sánchez, Pérez-López, y Anguera, 2014; Neuendorf, 2017), aunque con mayores cautelas metodológicas por un riesgo de subjetividad.

En los casos en que se optase en la misma investigación por utilizar, además de la observación sistemática, otra metodología (por ejemplo la selectiva, si se utilizasen técnicas de obtención de datos como el cuestionario o el test psicológico), en función de los respectivos objetivos específicos, no nos estaríamos refiriendo a los mixed methods por este motivo (aunque también pudiesen serlo, y los considerásemos como tal), sino a estudios multimethod (Anguera, Blanco-Villaseñor, Losada, SánchezAlgarra, et al., 2018).

En este trabajo nos interesa especialmente presentar una propuesta metodológica, que es novedosa y permite avanzar en la senda de la integración de los mixed methods. Se pretende constatar cómo el connecting permite fundamentar el quantitizing, lo cual consolida propuestas anteriores que han permitido constatar cómo la metodología observacional (directa e indirecta) se puede considerar como mixed method en sí misma (Anguera et al., 2017; Anguera y Hernández-Mendo, 2016), pero desde un planteamiento en donde no se combinan ni métodos ni técnicas, ni tampoco datos procedentes de diversas fuentes, sino que se transforma la información de forma diferente a como se describe en la literatura de los mixed methods. $\mathrm{Y}$, además, se enmarca, en las posibilidades que ofrece la perspectiva bayesiana, a partir de la propuesta de Onwuegbuzie et al. (2018).

Nuestra propuesta se asienta en una frase de Creswell \& Plano Clark $(2011$, p. 7) que valoramos especialmente: "There are three ways in which mixing occurs: merging or converging the two datasets by actually bringing them together, connecting the two datasets by having one build on the other, or embedding one dataset within the other so that one type of data provides a supportive role for the other dataset." (p. 7) (el resaltado es nuestro). Este mixing, en la opción de connecting, tomado tanto literalmente como desde una perspectiva más amplia, constituyen un punto de apoyo central para realizar un replanteamiento del quantitizing.

En efecto, desde la literalidad, el "connecting the two datasets by having one build on the other", implicará que una base de datos -que es de naturaleza cualitativa- puede dar lugar a otra mediante su transformación. Dicha transformación deberá garantizar el mantenimiento de su calidad informativa, aunque modificándose la apariencia. Y desde una perspectiva más amplia, el connecting permite la alternancia de etapas QUAL-QUAN-QUAL, que legitima el planteamiento genérico de los mixed methods, en tanto que se logra una integración total entre elementos cualitativos y cuantitativos. Con este replanteamiento podemos garantizar una forma innovadora para materializar 
nuestra propuesta de quantitizing, y que se materializará de forma clara en la observación sistemática.

Pero, además, tenemos que partir de la constatación de que la tradicional disyunción entre los planteamientos cualitativo y cuantitativo es un hecho, aunque en los últimos años ha ido calando la existencia de más opciones intermedias, que se han mostrádo en un amplio espectro de forma gradual. En este sentido, Bazeley $(2017,2018)$ considera que los planteamientos cualitativos y cuantitativos deberían considerarse como los extremos de un continuum, en el cual se va ubicando cada estudio de acuerdo con sus características específicas. Este planteamiento implica no solamente un cambio conceptual, sino que indudablemente tiene implicaciones metodológicas, y por ejemplo, en este sentido, Onwuegbuzie et al. (2018) abren una nueva vía de integración, que pretenden que sea total, y consideran que se puede lograr desde un planteamiento bayesiano, teniendo en cuenta la recogida de datos, su análisis y su interpretación, mediante la fórmula $1+1=1$, que es alternativa a la propuesta de Fetters and Freshwater (2015) que consideran la expresión $1+1=3$, que lo describen cuantitativamente como "qualitative + quantitative $=$ more than the individual components" (p. 116). ). En dicha dirección, Onwuegbuzie et al. (2018) generaron el acrónimo FIBT (Fully Integrated Bayesian Thinking) para referirse a este nuevo planteamiento de integración completa y dinamizaron las vías para lograrlo. En este sentido, Onwuegbuzie et al. (2018) consideran que esta integración total se puede lograr desde un planteamiento bayesiano, teniendo en cuenta la recogida de datos, su análisis y su interpretación, mediante la fórmula alternativa $1+1=1$.

Esta nueva visión implica la integración de diferentes tipos de datos, lo cual incluye la transformación de datos cualitativos en cuantitativos (Anguera, Camerino, et al., 2017; Sandelowski, Voils, \& Knafl, 2009), de datos cuantitativos en cualitativos (Onwuegbuzie \& Teddie, 2003; Sandelowski, et al., 2009), gráficos (Onwuegbuzie \& Dickinson, 2008), o cualquier otro tipo de información, de forma abarcadora ("crossover", en palabras de Onwuegbuzie \& Dickinson (2008)). Este planteamiento supone una gran esperanza de futuro, y estamos seguros de que aportará valiosos frutos, dado que permitirá una vía de integración completa (Onwuegbuzie \& Hitchcock, 2015). En palabras de Teddlie and Tashakkori (2009), "We believe that this [use of crossover mixed analyses] is one of the more fruitful areas for the further development of MM [mixed methods] analytical strategies" (p. 281), y se han seguido desarrollando, llevándolo incluso, entre otros, al ámbito de la comunicación no verbal (Hitchcock \& Onwuegbuzie, 2020). 


\section{RESULTADOS DE LA APLICACIÓN DE LA PROPUESTA DE QUANTITIZING MEDIANTE TRANSFORMACIÓN DE DATOS CUALITATIVOS EN DATOS TRATABLES CUANTITATIVAMENTE}

Queremos enfatizar la importancia y peso específico de una cadena de decisiones a adoptar muy responsablemente en los estudios de observación sistemática, y entre las cuales mencionamos los límites temporales del período de observación, el desglose en etapas específicos (cursos académicos, meses, trimestres, meses, semanas, sesiones, ...), el contexto (hogar, escuela, lugar de trabajo, espacio de entrenamientos deportivos, ...), agentes participantes (díada, tríada, participante central constante y variabilidad de los interactuantes, ...), obtención de la información (grabación magnética digital, grabación videográfica, fotografías, texto escrito, dibujos, ...), y, como pauta concreta en cada caso, todos aquellos aspectos o facetas que se requiera tomar en consideración.

Nuestra propuesta de quantitizing, adaptable a todas las situaciones que posibiliten la utilización de la metodología observacional como método científico (Anguera, BlancoVillaseñor, Losada, y Portell, 2018), se apoyan en el planteamiento inicial (Anguera, 2003), diseño observacional (Anguera et al., 2001), construcción de instrumento a medida (Anguera, Magnusson, y Jonsson, 2007), registro y codificación (Anguera y Blanco-Villaseñor, 2003), control de calidad del dato (Blanco-Villaseñor y Anguera, 2003), y análisis de datos (Blanco-Villaseñor, Losada, \& Anguera, 2003).

Hay que adoptar dos decisiones clave y construir un instrumento de observación. Las decisiones son las siguientes:

Primera decisión: Establecer las dimensiones del estudio (también denominados en la literatura científica niveles de respuesta o criterios). Pueden jerarquizarse, desplegándose (por ejemplo, macrodimensión / dimensión / subdimensión). Este concepto, relativo a las diferentes facetas o aspectos que se van a tener en cuenta, fue propuesto inicialmente por Weick (1968), y es altamente recomendable tener en cuenta el arco teórico. Incluimos dos ejemplos en Tabla 1:

Tabla 1. Ejemplo de dimensiones en dos estudios distintos correspondientes, respectivamente, a la interacción psicoterapéutica y a la comunicación verbal y no verbal entre terapeuta y paciente.

\begin{tabular}{|c|c|}
\hline $\begin{array}{lll}\begin{array}{l}\text { Dimensiones } \\
\text { psicoterapéutica }\end{array} & \text { en } & \text { interacción } \\
\end{array}$ & $\begin{array}{l}\text { Dimensiones en comunicación verbal y } \\
\text { no verbal entre terapeuta y paciente }\end{array}$ \\
\hline $\begin{array}{l}\text { Facilitating of conversation } \\
\text { Reflective function } \\
\text { Expressivity } \\
\text { Defensive expressions } \\
\text { Dislike } \\
\text { Ordering }\end{array}$ & $\begin{array}{l}\text { Verbal Mode-Structural Form (VeM-SF) } \\
\text { Verbal Mode-Communicative Intent (VeM-Cl) } \\
\text { Vocal Mode (VoM) }\end{array}$ \\
\hline
\end{tabular}

Ámbitos. Revista Internacional de Comunicación | ISSN: 1139-1979 | E-ISSN: 1988-5733, №. 49. (2020) 


\begin{tabular}{|c|c|}
\hline $\begin{array}{l}\text { Humor } \\
\text { Confrontation } \\
\text { Exclamation } \\
\text { Degradation of vocal behavior } \\
\text { Whispering } \\
\text { Touching } \\
\text { Noise } \\
\text { Surrounding noise } \\
\text { Silence }\end{array}$ & Interruption Mode (IM) \\
\hline \multicolumn{2}{|c|}{$\begin{array}{l}\text { "En ambos casos, de cada una de las dimensiones se han elaborado los sistemas de } \\
\text { categorías y se han propuesto, en su caso, los códigos que conforman el instrumento de } \\
\text { observación }\end{array}$} \\
\hline Fuente: Arias-Pujol and Anguera (2017, p. 4) & $\begin{array}{l}\text { Fuente: DelGiacco, Salcuni, and Anguera (2019, } \\
\text { Supplementary Material, p. 1-4) }\end{array}$ \\
\hline
\end{tabular}

La construcción del instrumento de observación se realiza a medida ( $a d$ hoc), y entre las opciones existentes -sistema de categorías, formato de campo, y formato de campo combinado con sistemas de categorías-, esta última es la que revela mayor aplicabilidad y se está utilizando con mayor profusión (Anguera et al., 2007). Consiste en plantear diversas macrodimensiones / dimensiones / subdimensiones (en función de la especificidad de cada caso), y para cada una de ellas [también se pueden denominar criterios / subcriterios] se construye una de las dos siguientes alternativas: a) Un sistema de categorías exhaustivo y mutuamente excluyente, si se dispone de marco teórico sobre aquella dimensión y existe una atemporalidad que garantice la necesaria exhaustividad; o b) un catálogo de conductas mutuamente excluyente que no requiere exhaustividad ni marco teórico y que es una lista abierta.

Segunda decisión: Proponer criterios de segmentación del continuum de la secuencia interactiva en sucesivas unidades de observación (Anguera, 2020, en prensa). La segmentación en unidades fue propuesta inicialmente por Dickman (1963) y Birdwhistell (1970), y desarrollada por Schegloff (2000) mediante el concepto de granularity.

Ambos conceptos (dimensión y unidad de observación) se han desarrollado posteriormente, tanto en observación directa (Anguera, 2017; Anguera \& Izquierdo, 2006; Izquierdo \& Anguera, 2018) como en indirecta (Anguera, 2017, 2020, en prensa), e integrado como piezas básicas para la preparación del instrumento observacional, necesario con el fin de codificar adecuadamente cada episodio.

Se incluyen dos ejemplos de instrumentos de observación en las Figuras 1 y 2, relativos, respectivamente, a la comunicación colaboración-oposición en un deporte y a un fragmento del instrumento de observación correspondiente a la evaluación de habilidades metacognitivas infantiles al resolver un puzzle: 


\begin{tabular}{|c|c|c|}
\hline No. & Dimensions & Categories (Codes) \\
\hline 1 & Ball possession & $\begin{array}{l}\text { PO) Possession of ball by team being observed; PC) Possession of ball by } \\
\text { opposing team; Inob) Unobservable }\end{array}$ \\
\hline 2 & Action initiation zone & $\begin{array}{l}\text { ZS10, ZS20, ZS30-safety sector; ZS40, ZS50, ZS60-creation sector; ZS70, ZS80, } \\
\text { ZS90-definition sector (see Figures 1-3) }\end{array}$ \\
\hline 3 & Action conclusion zone & $\begin{array}{l}\text { ZE10, ZE20, ZE30-safety sector; ZE40, ZF50, ZE60-creation section; ZE70, ZE80, } \\
\text { ZE90-definition sector. }\end{array}$ \\
\hline 4 & Contact with ball & $\begin{array}{l}\text { C1) single touch and regulatory throw-in/kick-in; C12) attempt to control the } \\
\text { ball with } 2 \text { or more touches resulting in loss of ball; C2) control of ball (including } \\
\text { picking up of ball by goalkeeper) followed by a pass or shot-regardless of } \\
\text { whether the ball reaches a team member or is recovered by an opponent; C23) } \\
\text { control of ball, followed by dribbling, and loss of ball; C24) control of ball, } \\
\text { followed by dribbling, attempt to go around one or more opponents, and loss of } \\
\text { ball; C3) control of ball, followed by dribbling and a pass or shot-regardless of } \\
\text { whether the ball reaches a team member or is recovered by an opponent; C4) } \\
\text { control of ball, followed by dribbling and passing of one or mone opponents, and } \\
\text { a pass or shot-regardless of whether the ball reaches a team member or is } \\
\text { recovered by an opponent; C5) Header. }\end{array}$ \\
\hline 5 & Interruptions & $\begin{array}{l}\text { FDFT) free kick for team being observed; FDFJ) offside for team being observed; } \\
\text { FFSB) throw-in for team being observed; FFSE) comer kick for team being } \\
\text { observed; FFSP) goal kick for team being observed; CDFT) free kick against team } \\
\text { being observed; CDFJ) offside against team being observed; (FFB) throw-in } \\
\text { against team being observed; (CFF) comer kick or goal kick against team being } \\
\text { observed; GF) goal scored by team being observed; GC) goal conceded by team } \\
\text { being observed; SN) neutral kick. }\end{array}$ \\
\hline 6 & Interceptions & $\begin{array}{l}\text { P) loss of ball; R) recovery of ball; IOC) Occasional interception with continuation } \\
\text { of play. }\end{array}$ \\
\hline 7 & Shot & $\begin{array}{l}\text { TG) shot resulting in goal; TI) shot intercepted by opponent other than the } \\
\text { goalkeeper; TM) shot between the posts not resulting in a goal; TF) shot wide of } \\
\text { the posts; TP) shot saved or cleared by the goalkeeper. }\end{array}$ \\
\hline
\end{tabular}

Figura 1. Instrumento de observación formado por formato de campo combinado con sistemas de categorías

Fuente: Amatria, Lapresa, Arana, Anguera, and Jonsson (2017, p. 4). 


\begin{tabular}{|c|c|c|c|c|c|}
\hline Criterion & Category systems & & Category description & Example & $\begin{array}{l}\text { Category } \\
\text { code }\end{array}$ \\
\hline \multirow[t]{3}{*}{ 1. Planning } & Inaccurate & & $\begin{array}{l}\text { The participant indicates where } \\
\text { he/she is going to place the plece but } \\
\text { he/she does it ambiguously and } \\
\text { vaguely, without using specific spatial } \\
\text { references (for example, without using } \\
\text { references of the type "up/down," "in } \\
\text { the roof area," "wall"). }\end{array}$ & $\begin{array}{l}\text { The participant says: "This (points to plece } \\
\text { 1) goes here (it indicates, without } \\
\text { precision, a large space in the upper area } \\
\text { of the table)". }\end{array}$ & plim \\
\hline & Accurate & & $\begin{array}{l}\text { The participant indicates in a concrete } \\
\text { and precise way where he/she is } \\
\text { going to place the plece and how, } \\
\text { using concrete spatial references on } \\
\text { what will be the location of the piece, } \\
\text { its orientation and/or its relationship } \\
\text { with the parts of the house. }\end{array}$ & $\begin{array}{l}\text { The participant says: "This (points to piece } \\
\text { 1) I will rotate and put it up here (points to } \\
\text { a specific space in the upper area of the } \\
\text { table), with this peak up (points to the right } \\
\text { angle of the piece), forming the roof of ling } \\
\text { house". }\end{array}$ & plp \\
\hline & Does not know/Does not answer & & $\begin{array}{l}\text { The participant does not give any } \\
\text { indication about how and where the } \\
\text { piece will be placed. }\end{array}$ & $\begin{array}{l}\text { The participant says: "I do not know how } \\
\text { the plece goes". }\end{array}$ & pln \\
\hline \multirow[t]{3}{*}{$\begin{array}{l}\text { 2. Planned or } \\
\text { moved plece }\end{array}$} & One & & $\begin{array}{l}\text { Plece of the puzzle marked with } \\
\text { number } 1\end{array}$ & $\begin{array}{l}\text { The participant says: "This piece the } \\
\text { participant points to the plece marked with } \\
\text { number (1) / will put it down". }\end{array}$ & uno \\
\hline & Two & & $\begin{array}{l}\text { Piece of the puzzle marked with } \\
\text { number } 2\end{array}$ & $\begin{array}{l}\text { The participant rotates the plece marked } \\
\text { with the number } 2 \text {. }\end{array}$ & dos \\
\hline & Three & & $\begin{array}{l}\text { Piece of the puzzle marked with } \\
\text { number } 3\end{array}$ & $\begin{array}{l}\text { The participant tries to place the piece } \\
\text { marked with number } 3 \text {. }\end{array}$ & tres \\
\hline 3. Monitoring & $\begin{array}{l}\text { According to the } \\
\text { planning }\end{array}$ & Success & $\begin{array}{l}\text { The participant places a plece } \\
\text { congruently to how he/she sald } \\
\text { he/she was going to do it before } \\
\text { starting the task, being this location of } \\
\text { the correct plece comparatively to the } \\
\text { house model to be built. }\end{array}$ & $\begin{array}{l}\text { The participant, during the planning, } \\
\text { pointing out plece 1, its sides and vertices } \\
\text { indicate that he/she will put the plece on } \\
\text { the top of the table, with the vertex of the } \\
\text { right angle upwards and the hypotenuse } \\
\text { parallel to the bottom edge of the table. } \\
\text { When he/she begins to perform the task, } \\
\text { takes piece } 1 \text { and places it as he/she said. } \\
\text { The result of this action is that piece } 1 \\
\text { remains as a roof of the house. }\end{array}$ & acpla \\
\hline
\end{tabular}

Figura 2. Fragmento de instrumento de observación formado por formato de campo combinado con sistemas de categorías Fuente: Escolano-Pérez, Herero-Nivela and Anguera (2019, p. 6).

Una vez se ha construido el instrumento de observación es posible efectuar el registro, que está formado por datos cualitativos, y que preferiblemente se estructurará como matriz de códigos.

En la Tabla 2 se presenta la estructura con que se conforma el registro, en formato de matriz de códigos que se obtendrá a partir de las dimensiones establecidas y la segmentación en unidades de observación, y siempre que se realice el registro a partir del parámetro orden o secuencia. Esta matriz resulta esencial para el proceso de quantitizing de los datos cualitativos (Anguera, en prensa; Anguera, Jonsson, \& Sánchez-Algarra, 2017; Anguera, Portell, Hernández-Mendo; Sánchez-Algarra, \& Jonsson, in press). La matriz de códigos tendrá por filas las unidades que se suceden en el tiempo, y por columnas las dimensiones. En consecuencia, la matriz de códigos resultante de volcar la realidad en el registro se forma a partir de la asignación de cada una de las unidades de observación al respectivo código. Debe entenderse, en 
consecuencia, que en cada fila de la tabla figurará la cadena de códigos correspondiente a las coocurrencias de cada una de las unidades de la conducta estudiada (ver ejemplo en Tabla 3).

Tabla 2. Estructura tabular preparada para obtener la matriz de códigos correspondiente al registro sistematizado

\begin{tabular}{|c|c|c|c|c|c|c|c|}
\hline & \multicolumn{6}{|c|}{$\begin{array}{c}\text { Dimensiones (también denominadas Criterios y Niveles de } \\
\text { respuesta) }\end{array}$} \\
\hline & & \multicolumn{2}{|c|}{ Dimensión 1} & \multirow{2}{*}{$\begin{array}{l}\text { Dimensión } \\
2\end{array}$} & \multicolumn{3}{|c|}{ Dimensión 3} \\
\hline & & $\begin{array}{l}\text { Subdimensión } \\
11\end{array}$ & $\begin{array}{l}\text { Subdimensión } \\
12\end{array}$ & & $\begin{array}{l}\text { Subdimensión } \\
31\end{array}$ & $\begin{array}{l}\text { Subdimensión } \\
32\end{array}$ & $\begin{array}{l}\text { Subdimensió } \\
\text { n } 33\end{array}$ \\
\hline \multirow{6}{*}{$\begin{array}{l}\text { og } \\
\frac{0}{0} \\
\frac{\pi}{0} \\
\frac{0}{c} \\
\frac{0}{c}\end{array}$} & $\begin{array}{l}\text { Unidad } \\
1\end{array}$ & Código & Código & Código & Código & Código & Código \\
\hline & $\begin{array}{l}\text { Unidad } \\
2\end{array}$ & Código & Código & Código & Código & Código & Código \\
\hline & $\begin{array}{l}\text { Unidad } \\
3\end{array}$ & Código & Código & Código & Código & Código & Código \\
\hline & $\begin{array}{l}\text { Unidad } \\
4\end{array}$ & Código & Código & Código & Código & Código & Código \\
\hline & Unidad & $\ldots$ & $\begin{array}{l}\cdots \\
\cdots\end{array}$ & $\ldots$ & $\ldots$ & $\ldots$ & $\ldots$ \\
\hline & & & & & & & \\
\hline
\end{tabular}

* Las columnas corresponden a las diversas dimensiones (con desplegamientos en subdimensiones) y las filas corresponden a las unidades resultantes de aplicar los criterios de segmentación, tanto en observación directa como indirecta. Las casillas de códigos incluirían los datos de un registro.

Fuente: Anguera (en prensa), y adaptado de Anguera, Portell, Hernández-Mendo, Sánchez-Algarra, \& Jonsson (in press)

\begin{tabular}{|c|c|c|c|c|c|}
\hline \multicolumn{6}{|c|}{ Tabla 3. Ejemplo simulado de registro } \\
\hline 112 & 121 & 23 & 313 & & 331 \\
\hline 111 & 122 & 23 & 311 & 322 & 333 \\
\hline 112 & 122 & 25 & 312 & & 331 \\
\hline 114 & 121 & & 312 & 323 & \\
\hline 114 & 121 & & 311 & & 332 \\
\hline
\end{tabular}

* Se ha elaborado de acuerdo a las dimensiones / subdimensiones y unidades indicadas en la Tabla

2, en formato de matriz de códigos

Fuente: Elaboración propia

Esta matriz de códigos constituye el esquema de cómo estará caracterizado el registro de los datos, todavía de carácter cualitativo (fase QUAL). En sentido figurado se requiere disponer, desde el inicio, de los pilares arquitectónicos y la nervadura de este "edificio". 
Por supuesto, en este planteamiento podemos igualmente incorporar, como elemento novedoso, la duración, y también diversas modalidades de datos como dibujos, sonidos, fotografías, etc., cuestión todavía incipiente, pero que encaja en los más avanzados planteamientos codificadores (Saldaña, 2013), los cuales, a su vez, hallan una lógica correspondencia en los apoyos informáticos con que contamos en la actualidad.

La codificación de registros (cualitativos) se realiza mediante programas informáticos libres como: GSEQ5 (http://bakeman.gsucreate.org/) (Bakeman \& Quera, 2011), HOISAN (www.menpas.com) (Hernández-Mendo, López-López, Castellano, MoralesSánchez, \& Pastrana, 2012), LINCE (http://lom.observesport.com) (Gabin, Camerino, Anguera, \& Castañer, 2012), LINCE PLUS (http://lom.observesport.com) (Soto, Camerino, Iglesias, Anguera, \& Castañer, 2019), o MOTS (www.menpas.com) (Castellano, Perea, Alday, \& Hernández-Mendo, 2008). Todos ellos permiten una gran eficacia en el quantitizing de registros cualitativos a partir del parámetro orden (sequence) (Bakeman, 1978) y posibilitan un potente análisis cuantitativo posterior.

Una vez ya disponemos de las matrices de códigos informatizadas, formadas únicamente por datos cualitativos, se nos abren amplísimas posibilidades para su análisis cuantitativo (fase QUAN). En trabajos anteriores (Anguera et al., 2017; BlancoVillaseñor et al., 2003) nos hemos pronunciado acerca de las muchas posibilidades existentes de análisis de datos procedentes de registros observacionales, y, más concretamente, de datos de naturaleza categórica o cualitativa, sea en algún ámbito específico (Anguera y Hernández Mendo, 2015; Escolano-Pérez y Blanco-Villaseñor, 2015), o de forma genérica. En una dirección convergente en varios aspectos, tenemos muy en cuenta, por su prestigio y relevancia, la propuesta de Ross \& Onwuegbuzie (2014), así como la más reciente de Hitchcock and Onwuegbuzie (2020), de elevado valor.

La información inicialmente cualitativa que se obtiene en el registro, y que es sumamente rica para el objetivo pretendido, permite unas amplias posibilidades de análisis cuantitativo (en muchos casos porque nos apoyamos en los parámetros primarios de orden y duración), que transforman los datos iniciales, y también cualitativo, integrándolos ambos.

Si consultamos la abundante documentación empírica existente en la actualidad, teniendo en cuenta el incremento exponencial de publicaciones empíricas sobre mixed methods que se evidencia en muchas revistas científicas, nos percatamos de que en su gran mayoría se aplican solamente técnicas analíticas convencionales (estadística descriptiva, correlaciones ordinales, aplicación de la teoría de la generalizabilidad, análisis de correspondencias, análisis de conglomerados, análisis de varianza, ...). 
Prestigiosos autores de mixed methods se han referido conceptualmente, y también técnicamente, a la cuantificación (Sandelowski, 2001; Sandelowski et al., 2009) y a todo lo que implica la transformación de los datos (Bazeley, 2009) (fase QUAN). Pero una gran diferencia de nuestra propuesta a partir de la metodología observacional (Portell, Anguera, Hernández-Mendo, \& Jonsson, 2015a), a favor de ésta, es que la cuantificación es más robusta, ya que no solamente se apoya en el recuento de ocurrencias de conducta, es decir, en la frecuencia, sino en los otros parámetros primarios (Bakeman, 1978; Bakeman y Gottman, 1989; Anguera et al., 2001; BlancoVillaseñor et al., 2003) de orden y duración, que entre ellos presentan un orden progresivo de inclusión. A partir del parámetro de la frecuencia se pueden efectuar análisis robustos que nos permiten conocer las relaciones de asociación entre diversas dimensiones de carácter categórico (Alsasua, Lapresa, Arana, \& Anguera, 2019; Losada, 2012), análisis de la variabilidad (Blanco-Villaseñor, Castellano, Hernández Mendo, Sánchez-López, y Usabiaga, 2014), o relaciones de causalidad entre dimensiones dicotómicas (Ardá, Maneiro, Rial, Losada, y Casal, 2014). Pero a partir de los parámetros de orden ( $\mathrm{y}$ en algunos casos también de duración) es posible desarrollar análisis cuantitativos robustos que consideramos de gran relevancia, como análisis secuencial de retardos (Bakeman y Gottman, 1989), análisis de coordenadas polares (Sackett, 1980), y detección de T-Patterns (Magnusson, 1996, 2000, 2020), dado que, desde diferentes perspectivas, nos permiten desvelar la estructura de los datos registrados, y en los últimos años hemos comprobado su creciente aplicabilidad, uso y resultados obtenidos, tanto individuamente como de forma complementaria. Para estos tres últimos tipos de análisis es imprescindible disponer del registro en formato de matriz de códigos, indicado anteriormente.

En definitiva, en nuestra propuesta la información inicialmente cualitativa que se obtiene en el registro, y que es sumamente rica para el objetivo pretendido, permite unas amplias posibilidades de análisis cuantitativo (debido básicamente a que nos apoyamos en los parámetros primarios de orden y duración), una vez transformados los datos iniciales, produciéndose la integración mediante el quantitizing, de acuerdo con nuestra propuesta.

La interpretación de los resultados obtenidos nos retorna a la etapa inicial de planteamiento del problema (fase QUAL).

\section{CONCLUSIONES}

Podemos afirmar que la metodología observacional se adapta perfectamente a la integración entre lo cualitativo y lo cuantitativo, ya que, simplificando drásticamente, siempre requerirá de la elaboración de un instrumento ad hoc a partir del cual se efectuará un registro (metodología cualitativa), y éste deberá someterse a un control de calidad y un análisis adecuado (metodología cuantitativa). Este punto central es de un considerable interés. 
Hemos desarrollado nuestro objetivo, mostrando una nueva forma de llevar a cabo el quantitizing, dentro del marco de los mixed methods, y a partir del interés por el connecting como vía clásica para la integración de elementos cualitativos y cuantitativos. Se destacan los beneficios que aporta la observación sistemática (directa e indirecta) en la obtención de datos cualitativos, los cuales se pueden sistematizar, transformándose en matrices de códigos a partir de las cuales se podrá operar cuantitativamente, utilizando técnicas de análisis más robustas que las utilizadas convencionalmente desde la estadística tradicional. El proceso de connecting se materializa en la sucesión de etapas QUAL-QUAN-QUAL, que fortalece aún más a la metodología observacional en el marco de los mixed methods.

Por otra parte, se está trabajando en la incorporación del análisis bayesiano en la integración entre elementos cualitativos y cuantitativos.

\section{Referencias}

Alsasua, R., Lapresa, D., Arana, J., \& Anguera, M. T. (2019). A log-linear analysis of efficiency in elite basketball applied to observational methodology. International Journal of Sports Science \& Coaching. Article first published online: March 25, 2019. https://doi.org/10.1177/1747954119837819

Amatria, M., Lapresa, D., Arana, J., Anguera, M. T., \& Jonsson, G. K. (2017). Detection and selection of behavioral patterns using Theme: a concrete example in grassroots soccer. Sports, 5, 20

Anguera, M. T. (1979). Observational Typology. Quality \& Quantity. European-American Journal of Methodology, 13(6), 449-484.

Anguera, M. T. (2000). Del registro narrativo al análisis cuantitativo: Radiografía de la realidad perceptible. En Ciencia $i$ cultura en el segle XXI. Estudis en homenatge a Josep Casajuana (pp. 41-71). Barcelona: Reial Academia de Doctors.

Anguera, M. T. (2003). Observational Methods (General). In R. Fernández-Ballesteros (Ed.), Encyclopedia of Psychological Assessment, Vol. 2 (pp. 632-637). London: Sage.

Anguera, M. T. (2017). Transiciones interactivas a lo largo de un proceso de desarrollo: Complementariedad de análisis. En C. Santoyo (Coord.), Mecanismos básicos de toma de decisiones: Perspectivas desde las ciencias del comportamiento y del desarrollo (pp. 179-213). México: CONACYT 178383/UNAM. I.S.B.N. 978-607-02-9426-6.

Anguera, M. T. (2020). Is It Possible to Perform "Liquefying" Actions in Conversational Analysis? The Detection of Structures in Indirect Observation. In L. Hunyadi and I. Szekrényes (Ed.), The Temporal Structure of Multimodal Communication (pp. 45-67). Intelligent Systems Reference Library, vol. 164. Springer Cham. DOI https://doi.org/10.1007/978-3-030-22895-8_3 
Anguera, M. T. (en prensa). Desarrollando la observación indirecta: Alcance, proceso, y habilidades metodológicas en el análisis de textos. En C. Santoyo y L. Colmenares (Coords.), Patrones de habilidades metodológicas y conceptuales de análisis, evaluación e intervención en ciencias del comportamiento. Ciudad de México: UNAM/PAPIIT, IN306715.

Anguera, M. T., y Blanco-Villaseñor, A. (2003). Registro y codificación en el comportamiento deportivo. En A. Hernández Mendo (Coord.), Psicología del Deporte (Vol. 2). Metodología (p. 6-34). Buenos Aires: Efdeportes (www.efdeportes.com).

Anguera, M. T., Blanco-Villaseñor, A., y Losada, J. L. (2001). Diseños Observacionales, cuestión clave en el proceso de la metodología observacional. Metodología de las Ciencias del Comportamiento, 3(2), 135-161.

Anguera, M. T., Blanco-Villaseñor, A., Losada, J. L., y Portell, M. (2018). Pautas para elaborar trabajos que utilizan la metodología observacional. Anuario de Psicología, 48, 9-17.

Anguera, M.T., Blanco-Villaseñor, A., Losada, J. L., y Sánchez-Algarra, P. (2019, Julio). Análisis del intercambio comunicativo: Planteamiento innovador del quantitizing. Congresso Ibero-Americano em Investigação Qualitativa (CIAIQ2019). Oporto.

Anguera, M. T., Blanco-Villaseñor, A., Losada, J. L., Sánchez-Algarra, P., \& Onwuegbuzie, A. J. (2018). Revisiting the Difference Between Mixed Methods and Multimethods: Is It All in the Name? Quality \& Quantity, 52, 2757-2770.

Anguera, M. T., Castañer, M., Portell, M., Sánchez-Algarra, P., \& Camerino, O. (in press). Bayesian Integration Applications in Physical Activity and Sport Research: Barriers and Solutions. In A.J. Onwuegbuzie and J. Hitchcook (Eds.), The Routledge Handbook for Advancing Integration in Mixed Methods Research. Abingdon, UK: Routledge.

Anguera, M. T., Camerino, O., Castañer, M., Sánchez-Algarra, P., \& Onwuegbuzie, A. J. (2017). The Specificity of Observational Studies in Physical Activity and Sports Sciences: Moving Forward in Mixed Methods Research and Proposals for Achieving Quantitative and Qualitative Symmetry. Frontiers in Psychology, 8:2196.

Anguera, M. T., \& Hernández-Mendo, A. (2015). Técnicas de análisis en estudios observacionales en ciencias del deporte. Cuadernos de Psicología del Deporte, 15(1), 13-30.

Anguera, M. T. y Hernández-Mendo, A. (2016). Avances en estudios observacionales en Ciencias del Deporte desde los mixed methods . Cuadernos de Psicología del Deporte, 16(1), 17-30.

Anguera, M. T., \& Izquierdo, C. (2006). Methodological approaches in human communication: From complexity of perceived situation to data analysis. In G. Riva, M.T. Anguera, B.K. Wiederhold \& F. Mantovani (Coord.), From Communication to Presence. Cognition, Emotions and Culture towards the Ultimate Communicative Experience (pp. 203-222). Amsterdam: IOS Press. 
Anguera, M. T., Jonsson, G. K., \& Sánchez-Algarra, P. (2017). Liquefying Text from Human Communication Processes: A Methodological Proposal Based on T-Pattern Detection. Journal of Multimodal Communication Studies, 4(1-2), 10-15.

Anguera, M. T., Magnusson, M. S., y Jonsson, G. K. (2007). Instrumentos no estándar. Avances en Medición, 5(1), 63-82.

Anguera, M. T., Portell, M., Chacón-Moscoso, S., \& Sanduvete-Chaves, S. (2018). Indirect observation in everyday contexts: Concepts and methodological guidelines within a mixed methods framework. Frontiers in Psychology, 9:13. doi: 10.3389/fpsyg.2018.00013

Anguera, M. T., Portell, P., Hernández-Mendo, A., Sánchez-Algarra, P., \& Jonsson, G. K. (in press). Diachronic analysis of qualitative data. In A.J. Onwuegbuzie and B. Johnson (Eds.), Reviewer's Guide for Mixed Methods Research Analysis. London: Routledge.

Ardá, T., Maneiro, R., Rial, A., Losada, J. L., y Casal, C. (2014). Análisis de la eficacia de los saques de esquina en la Copa del Mundo de Fútbol 2010. Un intento de identificación de variables explicativas. Revista de Psicología del Deporte, 23(1), 165-172.

Arias-Pujol, E. \& Anguera, M. T. (2017). Observation of interactions in adolescent group therapy: A mixed methods study. Frontiers in Psychology, 8:1188.

Bakeman, R. (1978). Untangling streams of behavior: Sequential analysis of observation data. In G.P. Sackett (Ed.), Observing Behavior, vol. 2 (pp. 63-78). Baltimore: University of Park Press.

Bakeman, R. \& Quera, V. (2011). Sequential analysis and observational methods for the behavioral sciences. Cambridge: Cambridge University Press.

Bazeley, P. (2009). Editorial: Integrating data analyses in mixed method research. Journal of Mixed Methods Research, 3(3), 203-207.

Bazeley, P. (2017). A mixed methods way of thinking and doing: integration of diverse perspectives in practice. Aoyama Journal of International Studies, 4, 17-28. doi: jairo.nii.ac.jp/0132/00041392/en

Bazeley, P. (2018). Integrating analysis in mixed methods research. London: Sage.

Bakeman, R. (1978). Untangling streams of behavior: Sequential analysis of observation data. In G.P. Sackett (Ed.), Observing Behavior, Vol. 2: Data collection and analysis methods (pp. 63-78). Baltimore: University of Park Press.

Bakeman, R. y Gottman, J. M. (1989) Observación de la interacción: Introducción al análisis secuencial. Madrid: Morata.

Birdwhistell, R. L. (1970). Kinesics and context: Essays and body motion communication. Philadelphia: University of Pennsylvania Press. 
Blanco-Villaseñor, A. (1997). Metodologies qualitatives en la investigació psicològica. Barcelona: Edicions de la Universitat Oberta de Catalunya.

Blanco-Villaseñor, A., y Anguera, M. T. (2003). Calidad de los datos registrados en el ámbito deportivo. En A. Hernández Mendo (Coord.), Psicología del Deporte (Vol. 2). Metodología (p. 35-73). Buenos Aires: Efdeportes (www.efdeportes.com).

Blanco-Villaseñor, A., Castellano, J., Hernández Mendo, A., Sánchez-López, C. R., y Usabiaga, O. (2014). Aplicación de la TG en el deporte para el estudio de la fiabilidad, validez y estimación de la muestra. Revista de Psicología del Deporte, 23(1), 131-137.

Blanco-Villaseñor, A., Losada, J. L., \& Anguera, M. T. (2003). Analytic techniques in observational designs in environment-behavior relation. Medio Ambiente $y$ Comportamiento Humano, 4(2), 111-126.

Castellano, J. Perea, A., Alday, L., \& Hernández-Mendo, A. (2008). The Measuring and Observation Tool in Sports. Behavior Research Methods, 40(3), 898-905.

Chacón-Moscoso, A., Anguera, M.T., Sanduvete-Chaves, S., Losada, J. L., Lozano-Lozano, J. A., \& Portell, M. (2019). Methodological quality checklist for studies based on observational methodology (MQCOM). Psicothema, 31(4), 458-464. doi: 10.7334/psicothema2019.116

Creswell, J. W., \& Plano Clark, V. L. (2017). Designing and conducting Mixed Methods Research (2nd ed., 2011). Thousand Oaks, CA: Sage.

Del Giacco, L., Salcuni, S., \& Anguera, M. T. (2019). The Communicative Modes Analysis System in Psychotherapy from Mixed Methods framework: Introducing a New Observation System for Classifying Verbal and Nonverbal Communication. Frontiers in Psychology, 10:782. doi: 10.3389/fpsyg.2019.00782

Dickman, H. R. (1963). The perception of behavioral units. In R. Barker (Ed.), The stream of behavior (pp. 23-41). New York: Appleton-Century-Crofts.

Escolano-Pérez, E. \& Blanco-Villaseñor, A. (2015). The longitudinal measurement of change: Intraindividual variability in behavior and interindividual differences observed in childhood. Anales de Psicología, 31(2), 545-551.

Escolano-Pérez, E., Herrero-Nivela, M. L., \& Anguera, M. T. (2019). Preschool metacognitive skill assessment in order to promote educational sensitive response from mixedmethods approach: complementarity of data analysis. Frontiers in Psychology, 10:1298. doi: 10.3389/fpsyg.2019.01298

Fetters M. D., \& Freshwater, D. (2015). The $1+1=3$ integration challenge. Journal of Mixed Methods Research, 9, 115-117.

Fuster-Parra, P., García-Más, A., Cantallops, J., Ponseti, F. J., \& Luo, Y. (2016). Ranking features on psychological dynamics of cooperative teams work through Bayesian networks. Symmetry 8:34. doi: 10.3390/sym8050034 
Fuster-Parra, P., García-Más, A., Ponseti, F. J., \& Leo, F. M. (2015). Team performance and collective efficacy in the dynamic psychology of competitive team: a Bayesian network analysis. Human Movement Science, 40, 98-118. doi: 10.1016/j.humov.2014.12.005

Fuster-Parra, P., García-Más, A., Ponseti, F. J., Palou, P., \& Cruz, J. (2013). A Bayesian network to discover relationships between negative features in sport. A case study of teen players. Quality and Quantity, 48, 1473-1491. doi: 10.1007/s11135-013-9848-y

Gabin, B., Camerino, O., Anguera, M. T., \& Castañer, M. (2012). Lince: multiplatform sport analysis software. Procedia - Social and Behavioral Sciences, 46, 4692-4694. doi: 10.1016/j.sbspro.2012.06.320

Hernández-Mendo, A., López-López, J. A., Castellano, J., Morales-Sánchez, V., and Pastrana, J. L. (2012). Hoisan 1.2: Programa informático para uso en metodología observacional. Cuadernos de Psicología del Deporte, 12, 55-78.

Hitchcock, J. H. \& Onwuegbuzie, A.J. (2020). Developing mixed methods crossover analysis approaches. Journal of Mixed Methods Research, 14(1), 63-83.

Hutt, S. J. \& Hutt, C. (1974). Direct observation and measurement of behavior. Sprinfield, II.: Charles C. Thomas.

Izquierdo, C., \& Anguera, M. T. (2018). Movement Notation Revisited: Syntax of the Common Morphokinetic Alphabet (CMA) System. Frontiers in Psychology, 9:1416.

Johnson, R. B., Onwuegbuzie, A. J., \& Turner, L. A. (2007). Toward a definition of mixed methods research. Journal of Mixed Methods Research, 1, 112-133.

Johnson, B. \& Turner, L. A. (2003). Data collection strategies in mixed methods research. In A. Tashakkori and Ch. Teddie (Eds.), Handbook of mixed methods in Social \& Behavioral Research (pp. 297-319). Thousand Oaks, Ca.: Sage.

Krippendorff, K. (2013). Content Analysis. An introduction to its methodology (3rd. ed.). Thousand Oaks, CA: Sage.

Losada, J. L. (2012). Relación entre zonas y contextos de interacción en el fútbol de alto rendimiento: Una aplicación multivariante. Motricidad. European Journal of Human Movement, 28, 171-183.

Magnusson, M. S. (1996). Hidden real-time patterns in intra- and inter-individual behavior: Description and detection. European Journal of Psychological Assessment, 12(2), 112123.

Magnusson, M. S. (2000). Discovering hidden time patterns in behavior: T-patterns and their detection. Behavior Research Methods, Instruments, \& Computers, 32(1), 93-110.

Magnusson, M. S. (2020). T-Pattern Detection and Analysis (TPA) With THEMETM: A Mixed Methods Approach. Frontiers in Psychology, 10:2663. doi: 10.3389/fpsyg.2019.02663 
Martin, P. \& Bateson, P. (1991). Measuring behavior: An introductory guide. New York, NY: Cambridge University Press.

Morales-Sánchez, V., Pérez-López, V., y Anguera, M. T. (2014). Tratamiento metodológico de la observación indirecta en la gestión de organizaciones deportivas. Revista de Psicología del Deporte, 23(1), 201-207.

Mucchielli, R. (1974). L'observation psychologique et psychosociologique. Paris: E.S.F.

Neuendorf, K. A. (2017). The content analysis. Guidebook. Thousand Oak, Ca.: Sage.

Olmedilla, A., Rubio, V. J., Fuster-Parra, P., Pujals, C., \& García-Más, A. (2018). A Bayesian Approach to Sport Injuries Likelihood: Does Player's Self-Efficacy and Environmental Factors Plays the Main Role? Frontiers in Psychology 9:1174. doi: 10.3389/fpsyg.2018.01174

Onwuegbuzie, A. J., \& Dickinson, W. B. (2008). Mixed methods analysis and information visualization: Graphical display for effective communication of research results. The Qualitative Report, 13, 204-225.

Onwuegbuzie, A. J., \& Hitchcock, J. H. (2015). Advanced mixed analysis approaches. In S. Hesse-Biber \& R. B. Johnson (Eds.), The Oxford handbook of multimethod and mixed methods research inquiry (pp. 275-295). New York, NY: Oxford University Press.

Onwuegbuzie, A. J., Hitchcock, J., Natesan, P., \& Newman, I. (2018). Using fully integrated thinking to address the $1+1=1$ integration challenge. International Journal of Multiple Research Approaches, 10(1), 666-678.

Onwuegbuzie, A. J., \& Teddlie, C. (2003). A framework for analyzing data in mixed methods research. In A. Tashakkori \& C. Teddlie (Eds.), Handbook of mixed methods in social and behavioral research (pp. 351-383). Thousand Oaks, CA: Sage.

Portell, M., Anguera, M. T., Chacón, S., \& Sanduvete, S. (2015). Guidelines for Reporting Evaluations based on Observational Methodology (GREOM). Psicothema, 27(3), 283289.

Portell, M., Anguera, M. T., Hernández-Mendo, A., \& Jonsson, G. K. (2015a). Quantifying biopsychosocial aspects in everyday contexts: an integrative methodological approach from the behavioral sciences. Psychology Research and Behavior Management, 8, 153160.

Portell, M., Anguera, M. T., Hernández-Mendo, A., \& Jonsson, G. K. (2015b). The legacy of Brunswik's representative design in the 21st century: methodological innovations for studying everyday life. The Brunswik Society Newsletter, 30, 38-40.

Ross, A., \& Onwuegbuzie, A. J. (2014). Complexity of quantitative analyses used in mixed research articles published in a flagship mathematics education journal. International Journal of Multiple Research Approaches, 8(1), 63-73. 
Sackett, G. P. (1980). Lag Sequential Analysis as a data reduction technique in social interaction research. En D.B. Sawin, R.C. Hawkins, L.O.Walker y J.H. Penticuff (Eds.), Exceptional infant. Phychosocial risks in infant-environment transactions (pp. 300-340). New York: Brunner/Mazel.

Saldaña, J. (2013). The coding manual for qualitative researchers. Los Angeles, CA: Sage.

Sánchez-Algarra, P. \& Anguera, M. T. (2013). Qualitative/quantitative integration in the inductive observational study of interactive behaviour: Impact of recording and coding predominating perspectives. Quality \& Quantity. International Journal of Methodology, 47(2), 1237-1257.

Sandelowski, M. (2001). Real qualitative researchers do not count: The use of numbers in qualitative research. Research in Nursing \& Health, 24(3), 230-240.

Sandelowski, M., Voils, C. I., \& Knafl, G. (2009). On quantitizing. Journal of Mixed Methods Research, 3, 208-222.

Schegloff, E. A. (2000). On granularity. Annual Review of Sociology, 26, 715-720.

Soto, A., Camerino, O., Iglesias, X., Anguera, M. T., \& Castañer, M. (2019). LINCE PLUS: Research software for behavior video analysis. Apunts. Educación Física y Deportes, 137, 149-153. doi: 10.5672/apunts.2014-0983.es.(2019/3).137.11

Teddie, C. \& Tashakkori, A. (2009). Foundations of mixed methods research. Integrating quantitative and qualitative approaches in the social and behavioral sciences. Thousand Oaks, Ca.: Sage.

Weick, K. E. (1968). Systematic observational methods. In G. Lindzey \& E. Aronson (Eds.), Handbook of Social Psychology (357-451), Vol. II. Reading, Mass.: Addison-Wesley.

*Los autores agradecen el apoyo del proyecto 'Vías de integración entre datos cualitativos y cuantitativos, desarrollo del caso múltiple, y síntesis como ejes principales para un futuro innovador en investigación de actividad física y deporte' [PGC2018-098742-B-C31] (20192021) (Ministerio de Ciencia, Innovación y Universidades de España / Agencia Estatal de Investigación / Fondo Europeo de Desarrollo Regional), que forma parte del proyecto coordinado 'New approach of research in physical activity and sport from mixed methods perspective' (NARPAS_MM) [SPGC201800X098742CV0]. Además, los autores agradecen el apoyo del Grupo Consolidado de Investigación de la Generalitat de Catalunya GRUP DE RECERCA I INNOVACIÓ EN DISSENYS (GRID). Tecnología i aplicació multimedia i digital als dissenys observacionals [Grant number 2017 SGR 1405].

*Durante el desarrollo de este artículo el Dr. Angel Blanco-Villaseñor falleció. Descanse en paz 